\title{
Expression of the promyelocytic leukemia zinc-finger in T-lymphoblastic lymphoma and leukemia has strong implications for their cellular origin and greater association with initial bone marrow involvement
}

\author{
Yoon K Jeon ${ }^{1,2}$, Heounjeong Go ${ }^{1,2}$, Soo J Nam ${ }^{1,2}$, Bhumsuk Keam ${ }^{3}$, Tae M Kim³ \\ Kyeong C Jung ${ }^{1}$, Hyoung J Kang ${ }^{4}$, Dong S Lee ${ }^{5}$, Joo R Huh ${ }^{6}$ and Seong H Park ${ }^{1}$ \\ ${ }^{1}$ Department of Pathology, Seoul National University Hospital, Seoul, South Korea; ${ }^{2}$ The Tumor Immunity \\ Medical Research Center, Cancer Research Center, Seoul National University College of Medicine, Seoul, \\ South Korea; ${ }^{3}$ Department of Internal Medicine, Seoul National University Hospital, Seoul, South Korea; \\ ${ }^{4}$ Department of Pediatrics, Cancer Research Institute, Seoul National University College of Medicine, Seoul, \\ South Korea; ${ }^{5}$ Department of Laboratory Medicine, Seoul National University Hospital, Seoul, South Korea \\ and ${ }^{6}$ Department of Pathology, Asan Medical Center, University of Ulsan College of Medicine, Seoul,
}

South Korea

\begin{abstract}
The promyelocytic leukemia zinc-finger (PLZF) is essential for the development of innate T cells (as represented by natural killer $T$ cells) for acquisition of their unique innate immune properties. We evaluated the PLZF protein expression in a variety of immature and mature lymphoid malignancies. PLZF was preferentially expressed in T-lymphoblastic lymphoma/acute lymphoblastic leukemia (T-LBL/ALL) in $\mathbf{5 0 \%}$ of the $\mathbf{5 4}$ cases. Among 51 cases of peripheral T-cell lymphoma not otherwise specified, only one (2\%) expressed PLZF. One mycosis fungoides case expressed PLZF in lymph node involved by tumor. Otherwise, PLZF was not detected in any other type of lymphoma. In T-LBL/ALL, PLZF expression was more common in CD4/CD8 double-negative (67\%) or CD8 single-positive subtypes (73\%) than in CD4/CD8 double-positive (13\%) and CD4 single-positive subtypes (0\%) $(P=0.001)$. Importantly, PLZF and CD1a expression were mutually exclusive in T-LBL/ALL $(P=0.001)$. This was also the case for T-cell receptor $\beta \mathrm{F} 1$ expression $(P=0.000)$. Most $(96 \%)$ of the PLZF-positive T-LBL/ALL cases showed initial bone marrow involvement compared with $39 \%$ of PLZF-negative cases $(P=0.000)$. Based on these findings, we suggest that T-LBL/ALLs that express PLZF arise from early immature double-negative thymocytes when the T-cell receptor $\beta$ chain has not yet expressed or innate T-cell precursors, and strongly imply bone marrow involvement.
\end{abstract}

Modern Pathology (2012) 25, 1236-1245; doi:10.1038/modpathol.2012.82; published online 4 May 2012

Keywords: innate T cells; lymphoma; natural killer T cells; promyelocytic leukemia zinc-finger; T lymphoblastic lymphoma/leukemia

The promyelocytic leukemia zinc-finger (PLZF), a transcription repressor of the BTB/POZ-ZF (broad

Correspondence: Dr YK Jeon, MD, PhD, Department of Pathology, Seoul National University Hospital, Yeongeon-dong 28, Jongro-gu, Seoul 110-744, South Korea.

E-mail; junarplus@chol.com

Received 13 January 2012; revised 7 March 2012; accepted 7 March 2012; published online 4 May 2012 complex, Tramtrack, Bric à brac (BTB) or poxvirus and zinc-finger (POZ)-zinc-finger (ZF)) family, was first identified as a fusion partner of retinoic acid receptor- $\alpha($ RAR- $\alpha)$ in a subset of acute promyelocytic leukemia having $t(11 ; 17)(q 23 ; q 21) .{ }^{1-3}$ PLZFRAR- $\alpha$ or RAR- $\alpha-$ PLZF fusion proteins function as dominant-negative molecules, contribute to leukemogenesis, and are associated with resistance to retinoid acid in acute promyelocytic leukemia. ${ }^{4-6}$ 
Under physiologic condition, PLZF is expressed in early hematopoietic stem cells and CD34 $(+)$ human bone marrow progenitor cells and its expression decreases during myeloid differentiation, and is thought to involve in the myeloid development and maintenance of hematopoietic stem cell population. ${ }^{7-11}$ PLZF also has a role in skeletal development and spermatogenesis, and it has a tumor-suppressive role in a few solid tumors. ${ }^{1,12-15}$

It was recently found that PLZF is essential for the development of natural killer T (NKT) cells and acquisition of the unique innate-like characteristics of innate T cells. ${ }^{16-19}$ Innate immunity is mediated by natural killer (NK) cells, monocytes/macrophages, and granulocytes. In general, this pathway enables the recognition of pathogens via germlineencoded pattern-recognition receptors (eg, the Toll-like receptor) and activation of the immediate immune response, and it does not involve immunological memory. Conversely, $\mathrm{B}$ and $\mathrm{T}$ cells are responsible for adaptive immunity. These cells have highly variable clonal immune receptors, namely immunoglobulin and T-cell receptors. ${ }^{20}$

The term 'innate T cells' refers to several different T-cell subsets that possess the properties of both innate and adaptive immune cells. They have unique features compared with conventional naive $\mathrm{T}$ cells, and share some characteristics with memory/effector T cells. After development and maturation in the thymus, innate $\mathrm{T}$ cells already have an activated or memory T-cell phenotype as an inherent property (eg, CD44 ${ }^{\text {hi }}$ ) and the ability to produce both Th1 (eg, IFN $\gamma$ ) and Th2 (eg, IL-4) cytokines immediately on stimulation, similar to memory T cells. ${ }^{21,22}$ PLZF is expressed in a variety of innate $\mathrm{T}$ cells and is implicated in their development, and it is now considered an innate T-cell determinant. ${ }^{19}$

This lineage-specific expression of PLZF in the immune system and lack of comprehensive information about PLZF expression in lymphoid malignancies led us to investigate the expression of PLZF in immature and mature lymphoid malignancies. Here, we demonstrated that PLZF is uniquely expressed in a substantial percentage of T-lymphoblastic lymphoma/acute lymphoblastic leukemia (T-LBL/ ALL). PLZF is thought to be a novel, relatively specific marker for T-LBL/ALL with respect to its cellular origin and early bone marrow involvement.

\section{Materials and methods}

\section{Patients and Tissue Samples}

Records of patients diagnosed with lymphoid malignancies at Seoul National University Hospital and Asan Medical Center were retrieved. All available materials were reviewed by two hematopathologists (YKJ and JRH) according to current World Health Organization criteria. The population of patients included in our study were summarized in Table 1. Ten samples of non-neoplastic tonsil or
Table 1 PLZF protein expression in lymphoid neoplasms

\begin{tabular}{|c|c|c|}
\hline Lymphoma type & $\mathrm{n}$ & $\begin{array}{c}\text { PLZF- } \\
\text { positive } \\
(\%)\end{array}$ \\
\hline T-lymphoblastic lymphoma/leukemia & 54 & $27(50 \%)$ \\
\hline B-lymphoblastic lymphoma/leukemia & 29 & 0 \\
\hline $\begin{array}{l}\text { Peripheral T-cell lymphoma not otherwise } \\
\text { specified }\end{array}$ & 51 & $1(2 \%)$ \\
\hline Angioimmunoblastic T-cell lymphoma & 46 & 0 \\
\hline Anaplastic large cell lymphoma, ALK+ & 15 & 0 \\
\hline Anaplastic large cell lymphoma, ALK- & 19 & 0 \\
\hline Extranodal NK/T-cell lymphoma, nasal type & 80 & 0 \\
\hline Enteropathy-associated T-cell lymphoma & $6^{\mathrm{a}}$ & 0 \\
\hline Primary cutaneous mature T-cell lymphoma & $13^{\mathrm{b}}$ & $1(8 \%)^{\mathrm{c}}$ \\
\hline $\begin{array}{l}\text { Chronic lymphocytic leukemia/small } \\
\text { lymphocytic lymphoma }\end{array}$ & 19 & 0 \\
\hline Mantle cell lymphoma & 23 & 0 \\
\hline Follicular lymphoma & 22 & 0 \\
\hline Diffuse large B-cell lymphoma & 58 & 0 \\
\hline $\begin{array}{l}\text { Extranodal marginal zone B-cell lymphoma } \\
\text { of mucosa-associated lymphoid tissue }\end{array}$ & 30 & 0 \\
\hline Nodal marginal zone B-cell lymphoma & 10 & 0 \\
\hline Burkitt lymphoma & 19 & 0 \\
\hline Hodgkin lymphoma & 66 & 0 \\
\hline Plasmacytoma & 28 & 0 \\
\hline
\end{tabular}

${ }^{\mathrm{a}} \mathrm{T}$-cell receptor $\beta \mathrm{F} 1$ immunohistochemistry staining was negative in three cases.

${ }^{\mathrm{b}} \mathrm{T}$-cell receptor $\beta \mathrm{F} 1$ staining was negative in four cases.

${ }^{\mathrm{C}} \mathrm{PLZF}$ staining was positive in lymph nodes involved in mycosis fungoides.

lymph nodes, and fetal and neonatal thymus were also examined as control. Clinical data including age, gender, white blood cell (WBC) count, mediastinal involvement, and bone marrow involvement at the time of presentation were recovered from medical records. The Institutional Review Board in Seoul National University Hospital approved this study.

\section{Immunohistochemistry}

For each patient, representative formalin-fixed paraffin-embedded tissue blocks were subjected to immunohistochemistry. Tissue microarrays were manufactured for diffuse large T-cell lymphoma, Hodgkin lymphoma, and a subset of T-LBL/ALL. Core tissues $(2 \mathrm{~mm}$ in diameter) were taken from individual paraffin blocks (donor blocks) and arranged into a new recipient paraffin block using a trephine apparatus. Immunohistochemical staining was performed using a Leica BOND-MAX automated immunostainer (Leica Microsystems, Wetzlar, Germany) and the following antibodies: PLZF (clone D-9, Santa Cruz Biotechnology, Santa Cruz, CA, USA), CD3 (clone F7.2.38, DakoCytomation, Copenhagen, Denmark), CD20 (clone L26, DakoCytomation), CD4 (clone 4B12, Thermo Fisher Scientific, Waltham, MA, USA), CD8 (clone SP16, Thermo Fisher Scientific), T-cell receptor 
$\beta$ F1 (clone 8A3, Thermo Fisher Scientific), TdT (polyclonal rabbit, DakoCytomation), and CD1a (clone MTB1, Novocastra Reagents, Leica Microsystems).

PLZF-specific staining intensity was graded as weak, moderate, or strong in the predominant pattern. The proportion of immunostained cells were classified as $0,1-25 \%, 26-50 \%, 51-75 \%$, or $>75 \%$.

\section{Statistical Analysis}

Either Pearson's $\chi^{2}$ test or Fisher's exact test was used to analyze differences between variables. $P$-values $<0.05$ were considered to be statistically significant, and all $P$-values were two sided. All statistical analyses were performed using SPSS version 19.0 for Windows (SPSS, Chicago, IL, USA). Overall survival was measured from the date of diagnosis to the date of death or to the date of the last follow-up visit. Event-free survival was defined as the date of diagnosis to the date of disease progression/relapse, death, or the date of the last follow-up. Survival analysis was performed using the Kaplan and Meier method using the log-rank test.

\section{Results}

\section{PLZF Expression in Lymphoid Malignancies}

PLZF was immunostained in the nucleus of lymphoid cells. In fetal and neonatal thymi, PLZFpositive cells were occasionally observed in the medulla and cortex (Figure 1a). With age, PLZFpositive cells decreased gradually in number to fewer than five per high-power field in the thymi and lymph nodes (Figure 1b).

The PLZF expression in lymphoid malignancies is summarized in Table 1. PLZF was preferentially expressed in T-LBL/ALL (50\%, 27/54 cases). Among 51 cases of peripheral T-cell lymphoma not otherwise specified, only $1(2 \%)$ expressed PLZF. One mycosis fungoides patient showed focal PLZF expression in a lymph node involved by tumor cells. Otherwise, PLZF expression was not detected in any cases of B-lymphoblastic lymphoma/leukemia, other mature T- or NK-cell lymphomas, mature T-cell lymphomas, Hodgkin lymphomas, or plasma cell neoplasm.

Although there were no PLZF-immunostained tumor cells in PLZF-negative lymphoid neoplasm, T-LBL/ALLs showed heterogeneous pattern of PLZF expression in terms of immunostaining intensity and the proportion of tumor cells (Figures 1c-f). As summarized in Table 2, 27 (50\%) of 54 T-LBL/ALL cases were completely negative for PLZF (Figure 1f). Eight $(15 \%)$ patients showed PLZF expression in $<25 \%$ of tumor cells (Figure 1e), and $17(32 \%)$ exhibited diffuse PLZF expression in more than half the tumor cells (Figure 1c). Among the 27 PLZFpositive T-LBL/ALLs, 25 cases showed moderate-tostrong PLZF staining intensity (Table 2).

\section{Immunophenotypes of T-LBL/ALL According to PLZF Expression}

T-LBL/ALL can be classified into diverse subtypes based on their developmental stages according to the expression of a panel of antigens. In brief, the pro- $\mathrm{T}$ and pre- $\mathrm{T}$ subtypes are negative for both CD4 and CD8 ('double-negative') and CD1a (-). Cortical $\mathrm{T}$ is positive for both CD4 and CD8 ('double-positive') and CD1a $(+)$. Medullary $\mathrm{T}$ expresses either CD4 or CD8 ('single-positive') and CD1a (-). ${ }^{23,24}$ Additionally, T-cell receptor $\alpha \beta$ expression is observed in late cortical and medullary T-cell lymphoblastic lymphoma/ leukemia. ${ }^{25}$

We examined the association between T-LBL/ALL subtype and PLZF expression. All T-LBL/ALL cases included in this study expressed cytoplasmic CD3 and at least one of the makers (eg, TdT, CD1a, CD99, or JL-1), indicating the precursor lymphoblastic nature. $^{23,26}$ As shown in Table 3, overall the double-negative subtype was most common (up to $45 \%$ ); followed by double-positive (28\%) and CD8 single-positive $(21 \%)$, and the CD4 single-positive subtype was least (6\%). CD1a was expressed in 9 $(17 \%)$ of 53 cases with different frequencies according to the T-LBL/ALL subtype $(P=0.002)$. None of the T-LBL/ALLs with double-negative subtype expressed CD1a, but 7 (47\%) of the 15 double-positive cases and each of the CD4 single-positive $(33 \%, 1 / 3)$ and CD8 single-positive $(9 \%, 1 / 11)$ cases were CD1a $(+)$ (data not shown). T-cell receptor $\beta \mathrm{F} 1$ was expressed in $14(28 \%)$ of 50 T-LBL/ALLs, and much more frequent in double-positive $(69 \%, 9 / 13)$ and CD4 single-positive $(67 \%, 2 / 3)$ subtypes compared with double-negative $(4 \%, 1 / 23)$ and CD8 singlepositive $(18 \%, 2 / 11)$ cases $(P=0.000)$ (data not shown). These expression patterns of CD1a and Tcell receptor $\beta \mathrm{F} 1$ were compatible with those expected.

PLZF expression differed significantly among the different T-LBL/ALL subtypes $(P=0.001)$. As summarized in Table 3, $16(67 \%)$ of the 24 doublenegative and $8(73 \%)$ of the 11 CD8 single-positive cases were PLZF-positive, whereas 2 (13\%) of the 15 double-positive and none of the 3 CD4 single-positive cases expressed PLZF. Moreover, CD1a and PLZF expression was mutually exclusive; namely, PLZF-positive T-LBL/ALLs were all negative for CD1a $(P=0.001)$. T-cell receptor $\beta \mathrm{F} 1$ and PLZF expression was also mutually exclusive $(P=0.000)$. Overall these data suggest that PLZF is frequently expressed in T-LBL/ALLs of early pro- or pre-T-cell features having doublenegative, CD1a $(-)$ and T-cell receptor $\beta \mathrm{F} 1(-)$ immunophenotypes. 

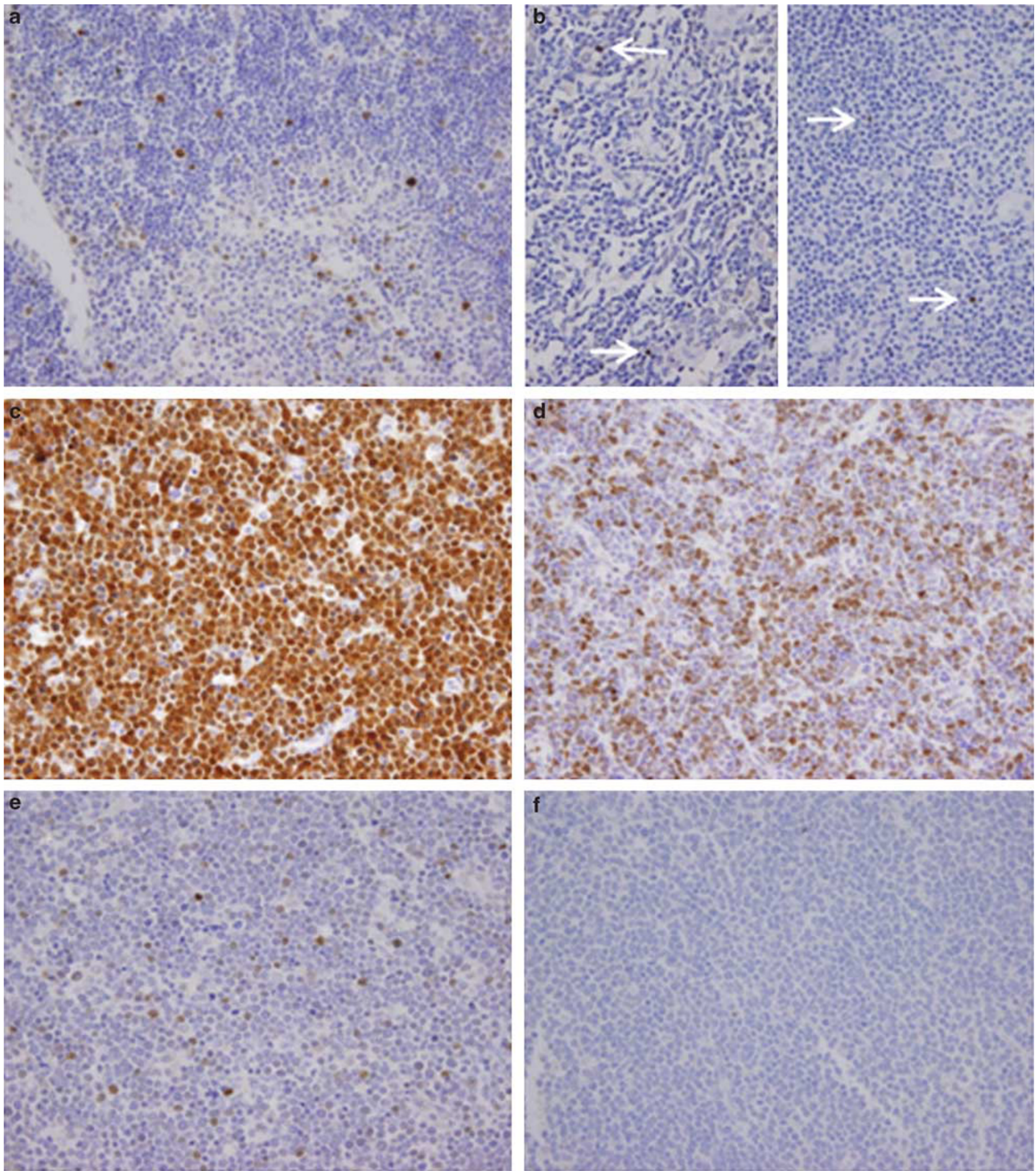

Figure 1 Immunohistochemical expression of PLZF in lymphoid tissue and neoplasms. PLZF was immunostained in the nucleus of lymphoid cells. (a) In a fetal thymus at gestational age 22 weeks, some scattered PLZF $(+)$ small to medium-sized cells were observed. In adult thymus (b, left) and lymph node (b, right), only a few small lymphoid cells were PLZF (+). T-LBL/ALLs showed variable expression of PLZF (c-f). Some reveal diffuse strong positivity in the tumor cells (c); others have weak-to-moderate intensity in some of the tumor cells (d); still others are completely negative for PLZF (f). (e) Occasionally, PLZF immunostained only in a small portion of tumor cells in T-LBL/ALL. (Original magnification, $\times 400$ in all).

\section{Clinical Features of PLZF-Positive T-LBL/ALL}

Clinical characteristics of T-LBL/ALLs according to PLZF expression are presented in Table 4. There were no statistical differences in age, gender,
WBC count, or mediastinal involvement between PLZF-positive and -negative T-LBL/ALLs.

Notably, PLZF expression in T-LBL/ALL was strongly associated with bone marrow involvement at presentation with $96 \%$ in PLZF-positive versus 
Table 2 PLZF expression patterns in T-lymphoblastic lymphoma/leukemia

\begin{tabular}{|c|c|c|c|c|c|c|}
\hline & \multicolumn{5}{|c|}{ Proportion of positive tumor cells } & \multirow[t]{2}{*}{ Total } \\
\hline & 0 & $1-25 \%$ & $26-50 \%$ & $51-75 \%$ & $>75 \%$ & \\
\hline \multicolumn{7}{|c|}{ Predominant staining intensity } \\
\hline None & 27 & 0 & 0 & 0 & 0 & $27(50 \%)$ \\
\hline Weak & 0 & 0 & 0 & 0 & 2 & $2(4 \%)$ \\
\hline Moderate & 0 & 7 & 0 & 2 & 4 & $13(24 \%)$ \\
\hline Strong & 0 & 1 & 2 & 1 & 8 & $12(22 \%)$ \\
\hline \multicolumn{7}{|l|}{ Total } \\
\hline 54 & $27(50 \%)$ & $8(15 \%)$ & $2(4 \%)$ & $3(6 \%)$ & $14(26 \%)$ & 54 \\
\hline
\end{tabular}

$39 \%$ of PLZF-negative cases $(P=0.000)$. This correlation was also significant when analyzed separately in children and adults. All of the four PLZF-positive versus one (14\%) of the seven PLZF-negative T-LBL/ ALLs had bone marrow involvement in childhood cases $(P=0.015)$. Among adult T-LBL/ALLs, $19(95 \%)$ of the 20 PLZF-positive cases had bone marrow involvement, whereas $8(50 \%)$ of the 16 PLZF-negative cases did $(P=0.003)$. About onefourth of the patients initially showed a leukemic presentation as defined by $>25 \%$ of blasts in bone marrow, and they had more frequent PLZF-positivity than the others. However the statistical

Table 3 Immunophenotypes of T-lymphoblastic lymphoma/leukemia according to PLZF expression

\begin{tabular}{|c|c|c|c|c|}
\hline & All cases n (\%) & PLZF-positive n (\%) & PLZF-negative n (\%) & $\mathrm{P}$-value \\
\hline \multicolumn{5}{|l|}{$C D 4$ and $C D 8$} \\
\hline Double-negative & $24 / 53(45 \%)$ & $16 / 26(62 \%)$ & $8 / 27(30 \%)$ & 0.001 \\
\hline Double-positive & 15/53 (28\%) & 2/26 (8\%) & 13/27 (48\%) & \\
\hline CD4 single-positive & $3 / 53(6 \%)$ & $0 / 26$ & $3 / 27(11 \%)$ & \\
\hline CD8 single-positive & $11 / 53(21 \%)$ & $8 / 26(31 \%)$ & $3 / 27(11 \%)$ & \\
\hline \multicolumn{5}{|l|}{$T d T$} \\
\hline Positive & 46/53 (87\%) & $23 / 26(89 \%)$ & $23 / 27(85 \%)$ & NS \\
\hline Negative & 7/53 (13\%) & $3 / 26(12 \%)$ & $4 / 27(15 \%)$ & \\
\hline \multicolumn{5}{|l|}{$C D 1 a$} \\
\hline Positive & 9/53 (17\%) & $0 / 26$ & 9/27 (33\%) & 0.001 \\
\hline Negative & $44 / 53(83 \%)$ & $26 / 26(100 \%)$ & $18 / 27(67 \%)$ & \\
\hline \multicolumn{5}{|l|}{ T-cell receptor $\beta$} \\
\hline Positive & $14 / 50(28 \%)$ & $0 / 25$ & $14 / 25(56 \%)$ & 0.000 \\
\hline Negative & $36 / 50(72 \%)$ & $25 / 25(100 \%)$ & $11 / 25(44 \%)$ & \\
\hline
\end{tabular}

Abbreviation: NS, not significant.

Table 4 Clinical features of T-lymphoblastic lymphoma/leukemia according to PLZF expression

\begin{tabular}{|c|c|c|c|c|}
\hline & All cases n (\%) & PLZF-positive n (\%) & PLZF-negative n (\%) & $\mathrm{P}$-value \\
\hline Age (yr), median (range) & $25.7(2-59)$ & $30.7(8-57)$ & $20(2-59)$ & \\
\hline Age (yr), mean \pm s.d. & $27.11 \pm 15.05$ & $30.67 \pm 13.21$ & $24.37 \pm 16.49$ & NS \\
\hline Age group & & & & NS \\
\hline Child $(\leqslant 15$ yr) & $14 / 54(26 \%)$ & $5 / 27(19 \%)$ & $9 / 27(33 \%)$ & \\
\hline Adult (>15 yr) & $40 / 54(74 \%)$ & $22 / 27(82 \%)$ & $18 / 27(67 \%)$ & \\
\hline Age $>35 \mathrm{yr}$ & $16 / 54(30 \%)$ & $11 / 27(41 \%)$ & $5 / 27(19 \%)$ & 0.074 \\
\hline Gender & & & & NS \\
\hline $\mathrm{M}$ & $38 / 54(70 \%)$ & $21 / 27(78 \%)$ & $17 / 27(63 \%)$ & \\
\hline $\mathrm{F}$ & $16 / 54(30 \%)$ & $6 / 27(22 \%)$ & $10 / 27(37 \%)$ & \\
\hline WBC $(/ \mu \mathrm{l})$, median $($ range,$\times 1000)$ & $6200(1-134)$ & $5800(1-134)$ & $6400(1.6-105)$ & \\
\hline WBC $(/ \mu \mathrm{l})$, mean \pm s.d. & $17585 \pm 29886$ & $20812 \pm 36391$ & $14478 \pm 22191$ & NS \\
\hline \multicolumn{5}{|l|}{ WBC group } \\
\hline$\geqslant 10^{4} / \mu \mathrm{l}$ & $14 / 53(26 \%)$ & $6 / 26(23 \%)$ & $8 / 27(30 \%)$ & NS \\
\hline \multicolumn{5}{|l|}{ Mediastinal involvement } \\
\hline Yes & $27 / 51(50 \%)$ & $11 / 23(48 \%)$ & $16 / 31(52 \%)$ & NS \\
\hline \multicolumn{5}{|l|}{ Bone marrow involvement } \\
\hline Yes & $32 / 47(68 \%)$ & $23 / 24(96 \%)$ & $9 / 23(39 \%)$ & 0.000 \\
\hline \multicolumn{5}{|l|}{ Relapse } \\
\hline Yes & $24 / 54(44 \%)$ & $13 / 27(48 \%)$ & $11 / 27(41 \%)$ & NS \\
\hline
\end{tabular}

Abbreviations: F, female; M, male; NS, not significant; s.d., standard deviation; yr, years. 
difference was insignificant (data not shown). Based on these data, it is highly probable that T-LBL/ALL expressing PLZF might have early bone marrow involvement by tumor cells of any quantity.

When the clinical variables were analyzed according to T-LBL/ALL phenotypes, mediastinal involvement was common among double-positive $(80 \%$, 12/15), CD4 single-positive $(67 \%, 2 / 3)$, or CD8 single-positive $(64 \%, 7 / 11)$ subtypes rather than double-negative $(21 \%, 5 / 24)(P=0.002)$. Bone marrow involvement was observed more frequently in cases of double-negative subtype (83\%, 20/24) compared with double-positive $(42 \%, 5 / 12)$ or single-positive $(60 \%, 6 / 10)(P=0.013)$. In addition, $26(81 \%)$ of 32 T-LBL/ALLs lacking T-cell receptor $\beta \mathrm{F} 1$ expression showed bone marrow involvement, whereas only $3(27 \%)$ of 11 T-cell receptor $\beta \mathrm{F} 1-$ expressing T-LBL/ALLs did $(P=0.002)$. Similarly, the absence of CD1a expression was significantly related with bone marrow involvement (74\% (29/39) in CD1a $(-)$ versus $29 \%(2 / 7)$ in CD1a $(+)$ T-LBL/ ALLs, $P=0.029$ ).

The relapse rate of T-LBL/ALL cases in this study was $44 \%$; this was similar between the
PLZF-positive and -negative groups. The 3-year event-free survival rate was $53 \%$ with a median of 21 months. Clinicopathologic factor that adversely affected patient event-free survival in the univariate analysis was age, and initial bone marrow involvement did with marginal significance (Figures 2a-c). However, PLZF expression showed no influence on the event-free survival or overall survival of T-LBL/ALL patients (Figure 2d).

\section{PLZF-Expressing Lymphoid Malignancies Other than T-LBL/ALL}

The clinicopathological features of the one PLZF $(+)$ peripheral T-cell lymphoma not otherwise specified case, which showed diffuse strong nuclear PLZF staining in the neoplastic cells (Figure 3c), are as follows. The patient was 73-year-old man presented with multiple systemic lymphadenopathy. Histologically, the lymph node architecture was diffusely effaced. Medium-to-large atypical lymphoid cells proliferated, infiltrated, and formed vague sheet-like patterns. The tumor cells had
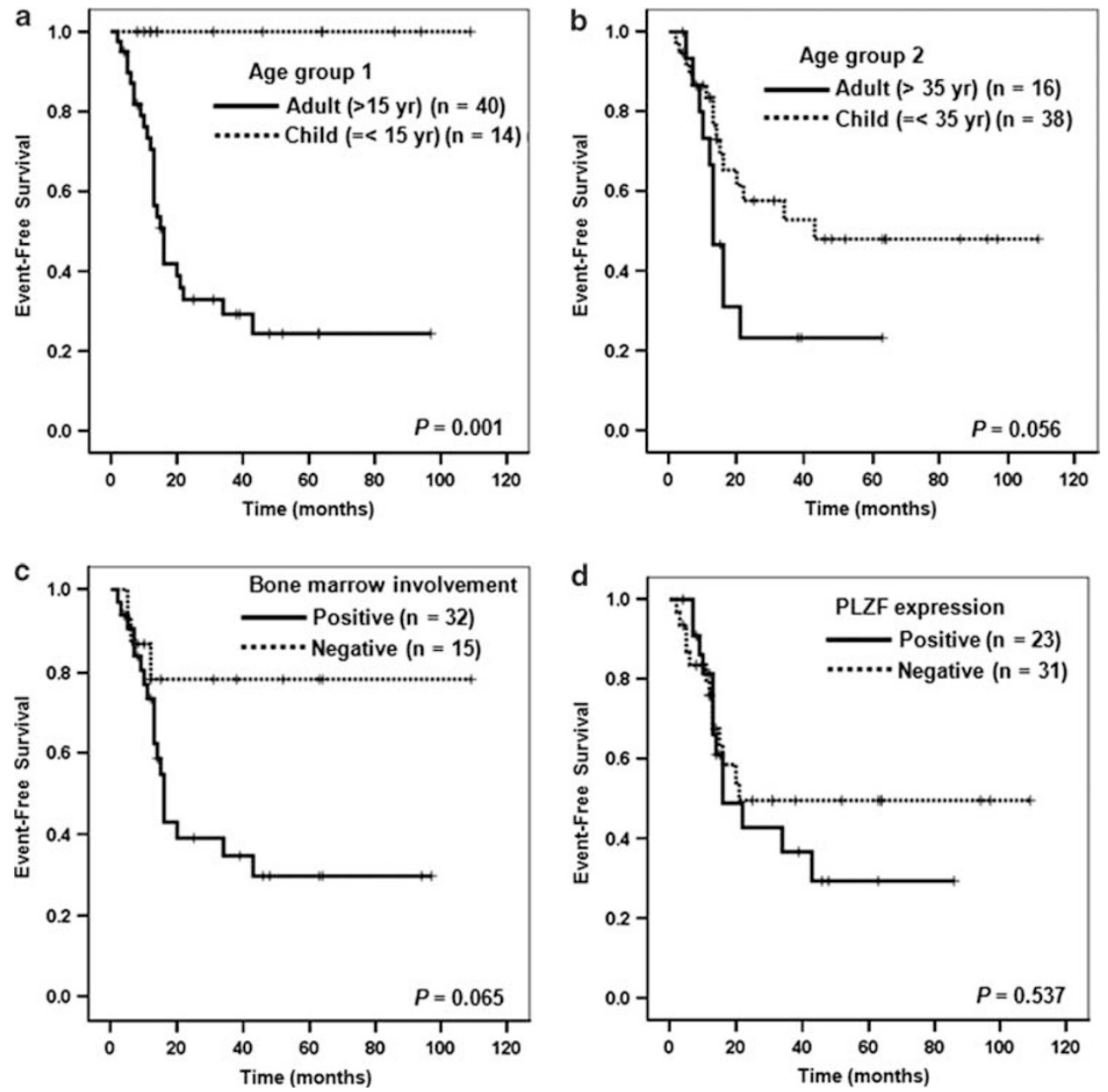

Figure 2 Plots for event-free survival of T-LBL/ALL patients according to the age (a, b), bone marrow involvement (c), or PLZF expression (d). Event-free survival time was calculated from the date of diagnosis to the date of disease progression/relapse, death, or the date of the last follow-up. Survival analysis was performed using the Kaplan and Meier method with the log-rank test. 

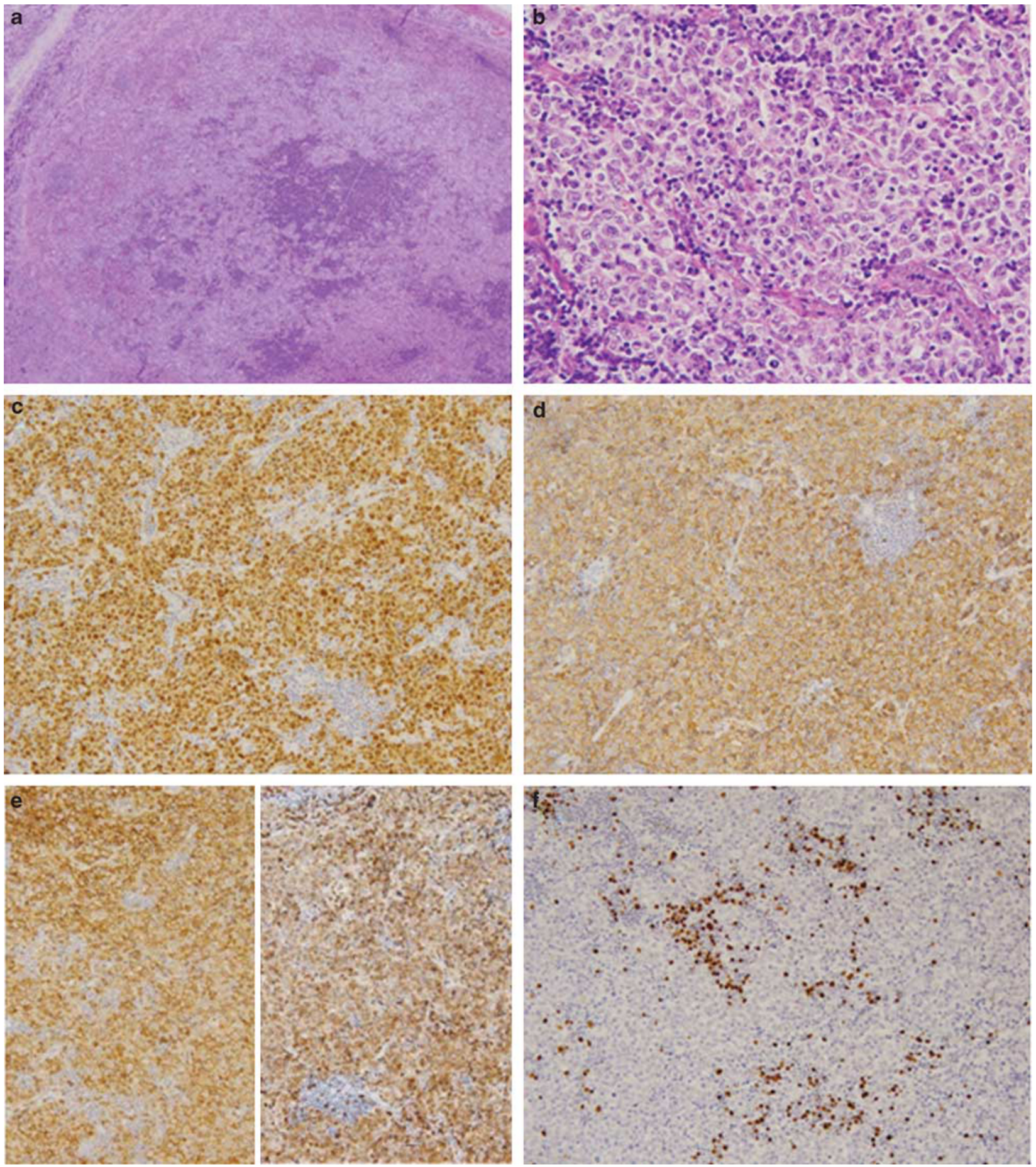

Figure 3 Pathologic features of the PLZF-positive peripheral T-cell lymphoma not otherwise specified case. (a) Medium-to-large atypical lymphoid cells diffusely effaced lymph node with a vague sheet-like pattern (H\&E, $\times 40)$. (b) Tumor cells have vesicular nuclei, grooved nuclear membrane, a few prominent nucleoli, and clear to eosinophilic cytoplasm (H\&E, $\times 400)$. In immunohistochemistry, tumor cells were positive for PLZF (c), CD3 (d), CD4 (e, left), T-cell receptor $\beta$ F1 (e, right). (f) EBV was infected in reactive B cells in in situ hybridization for EBV-encoded RNA.

vesicular nuclei with grooved nuclear membranes, small but distinct nucleoli, and clear to eosinophilic cytoplasm (Figures 3a and b). The tumor cells were positive for CD3, CD4, BCL2, and T-cell receptor $\beta \mathrm{F} 1$ (Figures 3c-e), and negative for CD8, CD56, CD30, CD10, granzyme B, and BCL6. T-cell monoclonality was observed by PCR analysis for T-cell receptor $\gamma$ gene rearrangement, and T-cell receptor $\mathrm{V} \alpha 24$ transcripts (as a determinant for invariant NKT cells) were not detected in reverse transcription (RT)-PCR analysis (data not shown). EBV in situ hybridization was positive in some reactive cells corresponding to 
B immunoblasts (Figure 3f). He died after 9 months due to disease progression despite of systemic chemotherapy.

In one patient with mycosis fungoides (a 54year-old man), focal moderate PLZF expression was detected in tumor cells infiltrating a lymph node (data not shown). The tumor cells were CD3 $(+)$, CD4 (+), T-cell receptor $\beta$ F1 $(+)$, and CD8 $(-)$. He received acitretin and interferon- $\alpha$ combination treatment, and was followed with variable symptoms for a year.

\section{Discussion}

In this study, we found that PLZF was preferentially and nearly exclusively expressed in T-LBL/ ALL (50\%) among lymphoid neoplasm using immunohistochemistry on formalin-fixed paraffinembedded tissues. We also encountered with a peculiar peripheral T-cell lymphoma not otherwise specified case $(2 \%)$ and mycosis fungoides case with lymph node involvement that expressed PLZF. Otherwise, PLZF expression was not detected in any cases of the B-lymphoblastic lymphoma/leukemia; other mature T, NK, B-cell, and plasma cell neoplasm; or Hodgkin lymphoma. The absence of PLZF expression in B-lymphoblastic lymphoma/ leukemia, the higher frequency of PLZF $(+)$ cases among T-LBL/ALL rather than CD1a, and its specific expression in lymphoma/leukemia of T-cell lineage compared with CD99 suggested that analysis of PLZF expression by immunohistochemistry could have some diagnostic value for determining the precursor $\mathrm{T}$ lymphoblastic nature of lymphoid malignancies, when used in combination with other markers.

PLZF is physiologically expressed in early hematopoietic progenitor cells and decreases in its level during differentiation into mature myeloid lineage. ${ }^{2,3,7,9,11}$ Recently, an intriguing role of PLZF in immune system has been described. ${ }^{16,17}$ Innate $\mathrm{T}$ cells bridges the innate and adaptive immune systems, including $\gamma \delta$ T cells and several subsets of $\alpha \beta$ T cells. In general, innate T cells (of an $\alpha \beta$ T-cell lineage) develop in the thymus from double-positive progenitors. These cells are selected by interactions with hematopoietic cells rather than thymic epithelial cells, and they express T-cell receptors restricted to non-classical MHC class Ib molecules rather than classical MHC class I or II molecules. They develop an activated and/or memory T-cell phenotype, and exhibit immediate effector functions along with cytokine secretion. ${ }^{21}$ Representative innate $\alpha \beta$ T-cell subsets in humans are CD1-restricted NKT and MR1 (MHC class I related)-specific mucosal-invariant $\mathrm{T}$ (MAIT) cells. NKT cells include type I NKT cells having invariant T-cell receptor (V $\alpha 24-\mathrm{J} \alpha 18$ and $\mathrm{V} \beta 11$ in humans), and type II (non-invariant) NKT cells that have more diverse T-cell receptor chains. ${ }^{27}$ PLZF is essential for the development of invariant NKT cells with acquisition of their unique innate-like properties in a mouse system. ${ }^{16,17}$ Recently, our group and others have reported a new distinct subset of innate $\alpha \beta$ T cells (T-T CD4 T cells), which are selected by MHC class II-expressing thymocytes and also depend on PLZF for their development. ${ }^{28-30}$ Moreover, we reported that the generation of T-T CD4 $\mathrm{T}$ cells is a physiological process in humans. ${ }^{18} \mathrm{Up}$ to now, PLZF has been exclusively expressed in a variety innate $\mathrm{T}$ cells including innate $\gamma \delta \mathrm{T}$ cells, type I and type II NKT cells, MAIT cells, and T-T CD4 T cells in lymphoid system. Therefore, PLZF expression is regarded as an innate T-cell determinant. ${ }^{19}$

The PLZF expression patterns in lymphoid malignancies observed in this study, in general, are rather reminiscent of the PLZF expression observed in the hematolymphoid system, and cast some important clues to understand the pathogenesis of $\mathrm{T}$ cell, particularly immature T-cell neoplasm. We in brief speculate that PLZF (+) T-LBL/ALLs suggest two possible cellular origins, that is, one from innate $\mathrm{T}$ cells and the other from early immature T-lymphoid precursors.

In terms of T-LBL/ALL subtypes based on T-cell development, PLZF expression was more common in the double-negative and CD8 single-positive subtypes rather than double-positive or CD4 singlepositive. PLZF expressions in a portion of doublenegative and CD8 single-positive T-LBL/ALLs were consistent with results from our previous study on PLZF expression in fetal human thymocytes. ${ }^{18}$ Although the question about what drives PLZF expression in immature cells committed to innate $\mathrm{T}$ cells remains to be answered, PLZF expression has been detected during double-negative stages of thymocytes. ${ }^{19}$ This is also consistent with our observation of PLZF positivity in double-negative T-LBL/ALLs. However, it was somewhat puzzling that there were no PLZF $(+)$ CD4 single-positive cases considering that up to $8 \%$ of fetal human CD4 single-positive thymocytes were positive for PLZF. ${ }^{18}$ A couple of points should be taken into consideration when interpreting this data. First, only a few (just three) cases of CD4 single-positive T-LBL/ALLs were included in this study. Second, neoplastic cells have the potential to aberrantly express any markers compared with normal counterparts.

Other important features of PLZF ( + ) T-LBL/ ALLs are mutually exclusive expressions between CD1a or T-cell receptor $\beta \mathrm{F} 1$ and PLZF, and strong association with initial bone marrow involvement. CD1a, a marker for double-positive cortical thymocytes, and T-cell receptor $\beta \mathrm{F} 1$, which expresses in late cortical and medullary thymocytes, were not found in any cases of PLZF $(+)$ T-LBL/ALL. It was reported that lack of T-cell receptor $\beta$ expression in cytoplasmic CD3 (+) T-acute lymphoblastic leukemias (ALLs) might represent immature T-ALLs of non-T-restricted precursor stage expressing immature markers such as CD34, CD13, and CD33, and 
predominantly with double-negative and CD1a (-) phenotype. ${ }^{31}$ PLZF expression was high in multipotent hematopoietic stem cells of bone marrow. ${ }^{11}$ Based on these notions, we think that PLZF $(+)$ T-LBL/ALLs might originate from thymocytes of early pro- or pre-T cell stage or more immature T-cell progenitors with double-negative phenotype and lacking $\mathrm{CD} 1 \mathrm{a}$ and $\mathrm{T}$-cell receptor $\beta \mathrm{F} 1$ expression, or immature $\gamma \delta \mathrm{T}$ cells. Therefore, purely on a theoretical basis, we suggest that a large number of T-LBL/ALL might in fact arise from double-negative stages of thymocytes and some of them aberrantly express either both CD4 and CD8 or single expression of CD4 and CD8 along their developmental process toward final maturation.

Of note, PLZF expression in NKT cells is elevated during the early stage of development and decreases as the cells mature. ${ }^{16,17}$ In humans, the number of PLZF ( +) thymocytes is high during the fetal stage and decreases in the postnatal period. ${ }^{18}$ In a mouse system where all CD4 $\mathrm{T}$ cells were designed to express PLZF molecule, PLZF was detected in a portion of T-T CD4 T cells. ${ }^{30}$ These observations suggest that although PLZF could be a specific marker for innate $\mathrm{T}$ cells, it is possible that many innate $\mathrm{T}$ cells gradually lose their PLZF expression when mature. Based on this, it is highly conceivable that PLZF expression in mature T-cell lymphomas is very rare and that the PLZF expression is frequent in T-LBL/ALLs in the double-negative phenotype, as in this study.

In this present study, only one peripheral T-cell lymphoma not otherwise specified and one mycosis fungoides patient expressed PLZF, but were negative for $\mathrm{V} \alpha 24$ according to RT-PCR (data not shown). Therefore, it is possible that these cases might represent a peripheral T-cell lymphoma of innate T-cell origin other than invariant NKT cells.

NK-cells, a representative type of innate lymphoid cells, are not known to express PLZF. ${ }^{19}$ Consistently, none of the extranodal NK/T-cell lymphomas that predominantly originated from NK cells showed PLZF expression.

In several solid tumors and myeloid cell lines, PLZF inhibited cell proliferation and induced apoptosis..$^{8,12,13,32}$ In this study, T-LBL/ALL patients with any bone marrow involvement showed shorter event-free survival, which was consistent with previous report. ${ }^{33}$ Although PLZF was a strong indicator for initial bone marrow involvement of any quantity in this study, PLZF expression itself had no influence on the survival of T-LBL/ALL patients. However, the heterogeneity of therapeutic modality restricted the accurate evaluation for prognostic implications of PLZF expression in T-LBL/ALLs.

In summary, we performed the first comprehensive investigation of the expression of PLZF, an innate T-cell determinant, in lymphoid malignancies. We showed that this protein is uniquely expressed in a considerable portion of T-LBL/ALL, but rarely in peripheral T-cell lymphomas. PLZF expression in T-lymphoma/leukemia suggests a possible origin in immature lymphocytes of innate T-cell lineage or early T-lymphoid precursors, and has important clinical implication regarding a greater probability of early bone marrow involvement.

\section{Acknowledgement}

This work was supported by a grant from the Seoul National University Hospital Research Fund (20112061)

\section{Disclosure/conflict of interest}

The authors declare no conflict of interest.

\section{References}

1 Kelly KF, Daniel JM. POZ for effect-POZ-ZF transcription factors in cancer and development. Trends Cell Biol 2006;16:578-587.

2 Chen SJ, Zelent A, Tong JH, et al. Rearrangements of the retinoic acid receptor alpha and promyelocytic leukemia zinc finger genes resulting from $t(11 ; 17)$ (q23;q21) in a patient with acute promyelocytic leukemia. J Clin Invest 1993;91:2260-2267.

3 Chen Z, Brand NJ, Chen A, et al. Fusion between a novel Kruppel-like zinc finger gene and the retinoic acid receptor-alpha locus due to a variant $t(11 ; 17)$ translocation associated with acute promyelocytic leukaemia. EMBO J 1993;12:1161-1167.

4 Rego EM, Ruggero D, Tribioli C, et al. Leukemia with distinct phenotypes in transgenic mice expressing PML/RAR alpha, PLZF/RAR alpha or NPM/RAR alpha. Oncogene 2006;25:1974-1979.

5 Guidez F, Parks S, Wong H, et al. RARalpha-PLZF overcomes PLZF-mediated repression of CRABPI, contributing to retinoid resistance in $\mathrm{t}(11 ; 17)$ acute promyelocytic leukemia. Proc Natl Acad Sci USA 2007;104:18694-18699.

6 Rice KL, Hormaeche I, Doulatov S, et al. Comprehensive genomic screens identify a role for PLZF-RARalpha as a positive regulator of cell proliferation via direct regulation of C-MYC. Blood 2009;114: 5499-5511.

7 Dick JE, Doulatov S. The role of PLZF in human myeloid development. Ann NY Acad Sci 2009;1176: 150-153.

8 Shaknovich R, Yeyati PL, Ivins S, et al. The promyelocytic leukemia zinc finger protein affects myeloid cell growth, differentiation, and apoptosis. Mol Cell Biol 1998;18:5533-5545.

9 Doulatov S, Notta F, Rice KL, et al. PLZF is a regulator of homeostatic and cytokine-induced myeloid development. Genes Dev 2009;23:2076-2087.

10 Spinello I, Quaranta MT, Pasquini L, et al. PLZFmediated control on c-kit expression in CD34(+) cells and early erythropoiesis. Oncogene 2009;28: $2276-2288$.

11 Reid A, Gould A, Brand N, et al. Leukemia translocation gene, PLZF, is expressed with a speckled nuclear 
pattern in early hematopoietic progenitors. Blood 1995;86:4544-4552.

12 Cheung M, Pei J, Pei Y, et al. The promyelocytic leukemia zinc-finger gene, PLZF, is frequently downregulated in malignant mesothelioma cells and contributes to cell survival. Oncogene 2010;29:1633-1640.

13 Felicetti F, Errico MC, Bottero L, et al. The promyelocytic leukemia zinc finger-microRNA-221/-222 pathway controls melanoma progression through multiple oncogenic mechanisms. Cancer Res 2008;68: 2745-2754.

14 Barna M, Hawe N, Niswander L, et al. Plzf regulates limb and axial skeletal patterning. Nat Genet 2000;25:166-172.

15 Costoya JA, Hobbs RM, Barna M, et al. Essential role of PLZF in maintenance of spermatogonial stem cells. Nat Genet 2004;36:653-659.

16 Savage AK, Constantinides MG, Han J, et al. The transcription factor PLZF directs the effector program of the NKT cell lineage. Immunity 2008;29:391-403.

17 Kovalovsky D, Uche OU, Eladad S, et al. The BTB-zinc finger transcriptional regulator PLZF controls the development of invariant natural killer T cell effector functions. Nat Immunol 2008;9:1055-1064.

18 Lee YJ, Jeon YK, Kang BH, et al. Generation of PLZF+ CD4+ T cells via MHC class II-dependent thymocytethymocyte interaction is a physiological process in humans. J Exp Med 2010;207:237-246.

19 Alonzo ES, Sant'Angelo DB. Development of PLZFexpressing innate $\mathrm{T}$ cells. Curr Opin Immunol 2011;23:220-227.

20 Janeway Jr CA, Medzhitov R. Innate immune recognition. Annu Rev Immunol 2002;20:197-216.

21 Berg LJ. Signalling through TEC kinases regulates conventional versus innate CD8(+) T-cell development. Nat Rev Immunol 2007;7:479-485.

22 Van Kaer L. alpha-Galactosylceramide therapy for autoimmune diseases: prospects and obstacles. Nat Rev Immunol 2005;5:31-42.

23 Borowitz MJ, Chan JKC. Precursor lymphoid neoplasm. In: Swerdlow SH, Campo E, Harris NL, Jaffe ES, Pileri SA, Stein H, et al. (eds). WHO Classification of Tumours of Haematopoietic and Lymphoid Tissues 4th edn International Agency for Research on Cancer: Lyon, 2008, pp 167-178.

24 Han X, Bueso-Ramos CE. Precursor T-cell acute lymphoblastic leukemia/lymphoblastic lymphoma and acute biphenotypic leukemias. Am J Clin Pathol 2007;127:528-544.

25 Racke FK, Borowitz MJ. Precursor B- and T-cell neoplasms. In: Jaffe ES, Harris NL, Vardiman JW, Campo E, Arber DA (eds). Hematopathology 1st edn Elsevier: St Louis, MO, 2011, pp 629-639.

26 Shin YK, Choi EY, Kim SH, et al. Expression of leukemia-associated antigen, JL1, in bone marrow and thymus. Am J Pathol 2001;158:1473-1480.

27 Godfrey DI, MacDonald HR, Kronenberg M, et al. NKT cells: what's in a name? Nat Rev Immunol 2004;4: 231-237.

28 Choi EY, Jung KC, Park HJ, et al. Thymocyte-thymocyte interaction for efficient positive selection and maturation of CD4 T cells. Immunity 2005;23:387-396.

$29 \mathrm{Li} \mathrm{W,} \mathrm{Kim} \mathrm{MG,} \mathrm{Gourley} \mathrm{TS,} \mathrm{et} \mathrm{al.} \mathrm{An} \mathrm{alternate} \mathrm{pathway}$ for CD4 T cell development: thymocyte-expressed MHC class II selects a distinct $\mathrm{T}$ cell population. Immunity 2005;23:375-386.

30 Lee YJ, Jung KC, Park SH. MHC class II-dependent T-T interactions create a diverse, functional and immunoregulatory reaction circle. Immunol Cell Biol 2009;87:65-71.

31 Asnafi V, Beldjord K, Boulanger E, et al. Analysis of TCR, pT alpha, and RAG-1 in T-acute lymphoblastic leukemias improves understanding of early human T-lymphoid lineage commitment. Blood 2003;101: 2693-2703.

32 Brunner G, Reitz M, Schwipper V, et al. Increased expression of the tumor suppressor PLZF is a continuous predictor of long-term survival in malignant melanoma patients. Cancer Biother Radiopharm 2008;23:451-459.

33 Coustan-Smith E, Sandlund JT, Perkins SL, et al. Minimal disseminated disease in childhood T-cell lymphoblastic lymphoma: a report from the children's oncology group. J Clin Oncol 2009;27:3533-3539. 